\title{
Review of: "Ultrahigh-speed graphene-based optical coherent receiver"
}

\author{
Sezer Erkilinc ${ }^{1}$
}

1 Infinera

Potential competing interests: The author(s) declared that no potential competing interests exist.

Considering the exponentially increasing demand for bandwidth in the digital society that we live in, this paper timely proposes an integrated optical coherent receiver (ICR) based on a 90-degree optical hybrid and graphene-on-plasmonic slot waveguide photodetectors, exhibiting a compact footprint and a high bandwidth, $67 \mathrm{GHz}$. It is anticipated that coherent technologies will be utilized more and more as the fibre penetrates deeper in telecommunication networks.

The design, characteristics and performance test for the proposed ICR have presented clearly and answer many questions who would be interested in such a device. The graphene-based ICRs seem quite promising from a bandwidth-performance trade-off standpoint. Furthermore, the authors sound optimistic extending the results reported here from $67 \mathrm{GHz}$ to $150 \mathrm{GHz}$ which would be significant advancement for the field.

I would raise two comments that the authors can consider in their following studies. 1) Although a comparison in terms of packaging is presented in Supplementary Note 4 and Supplementary Table 2 with the state-of-the-art (InP- and Silicon-based) ICRs, it would have been very insightful to also compare their performance at the same bit rate to observe the gap between these established technologies versus the disruptive technology. 2) I would highly suggest that the authors would focus on the realization of PDM16/64-QAM signalling scheme(s) in their following graphene-based OCR work since they are the industry standards in metro and aggregation networks (over 80 to $120 \mathrm{~km}$ links). 